Revista Calidad en la Educación Superior

Programa de Autoevaluación Académica

Universidad Estatal a Distancia, Costa Rica

ISSN 1659-4703

revistacalidad@uned.ac.cr

\title{
LA PARTICIPACIÓN INFANTIL Y LA CONVIVENCIA EN ESPACIOS ESCOLARES: UNA EXPERIENCIA PARA SU MEJORA EN LA EDUCACIÓN PRIMARIA
}

\author{
CHILD PARTICIPATION AND LIVING TOGETHER IN SCHOOL SPACE: AN EXPERIENCE \\ OF IMPROVEMENT IN PRIMARY EDUCATION.
}

\author{
Denys Serrano Arenas 1 \\ dehus_res@hotmail.com \\ Liberio Victorino Ramírez2 \\ victorinoramrezliberio@yahoo.com.mx \\ Universidad Autónoma Chapingo, México
}

Volumen 6, número 1

Mayo 2015

Pp. $76-90$

Recibido: 25 de mayo, 2014

Aprobado: 5 de marzo, 2015

\footnotetext{
${ }^{1}$ Denys Serrano Arenas, estudiante del Doctorado en Ciencias en Educación Agrícola Superior en la Universidad Autónoma Chapingo, docente en Educación Básica y participante en cuerpos académicos en Educación y Sustentabilidad Ambiental. Correo electrónico: dehus_res@hotmail.com

2Liberio Victorino Ramírez, profesor e investigador de la Universidad Autónoma Chapingo, adscrito al Departamento de Sociología Rural. Miembro del Sistema Nacional de Investigadores (SNI). Director de grupos académicos a nivel nacional e internacional, con más de quince libros publicados. Correo electrónico:victorinoramrezliberio@yahoo.com.mx
} 


\section{Resumen}

Es prioritario brindarles medios a las niñas y los niños para cambiar su autoconcepción de sujetos a agentes de cambio, que no solo viven conforme a las condiciones que les tocó vivir, sino que tienen el poder colectivo de cambiar su entorno a través de la acción organizada. Para ello, la participación es un bien esencial, un derecho de las niñas y los niños que permite vincular a las personas a través de la expresión de intereses, preocupaciones y deseos.

Partiendo de la implementación de un programa de participación infantil, se realizó la presente investigación con un grupo escolar, el cual, permitió conocer la relación entre el aumento de la responsabilidad con su entorno y el interés de seguir realizando prácticas participativas.

Palabras claves: participación, valores, acción y niñez

\section{Abstract}

It's a priority to give girls and boys means to change their self-conception from subjects to agents of change, that they not only live according to the conditions they live in, but that they have the collective power to change their environment through organized action. The participation is an essential good, a right for girls and boys, which allows them to gather people through the expression of interests, preoccupations and desires.

Starting from an implementation of a child participatory program, the present investigation was realized with a school group, which allowed us to know the relationship between the increase of responsibility with their environment and the interest to keep having a participatory practice.

Keywords: participation, values, action and citizen.

Cuando se habla de sociedades democráticas un indicador trascendente es la participación de las personas en la toma de decisiones que les afectan, así como la generación de redes sociales por parte de una comunidad para la resolución de los problemas de su entorno; lo anterior implica a personas que se visualizan como actores en la transformación de su comunidad.

La participación social se vincula con el involucramiento - con compromiso, cooperación y responsabilidad — de las personas para el logro de objetivos comunes. Esta implica compartir 
responsabilidades y una articulación establecida y definida entre intereses comunes y problemáticas percibidas por los y las participantes (Chávez, 2006).

A pesar de su importancia y trascendencia, México tiene índices bajos en su ejercicio, esto se muestra en la encuesta de la World Values Survey (2005), en la que se registra el $16 \%$ de confianza interpersonal, por debajo de un promedio de $28 \%$ registrado en 56 países. Esta información se obtuvo a partir de dos cuestionamientos principales: el primero se refiere a los niveles de confianza que sienten por los miembros de su comunidad y el segundo a los niveles de confianza que sienten por una persona en particular. A partir de este estudio se realizó la Encuesta de capital social urbano 2006 (SEDESOL, 2007), que señala que el 70\% de las personas considera importante cuidarse de la gente que la rodea; no confiar en otros miembros de la comunidad, según el pueblo mexicano, es la razón principal por la que las personas no se ayudan. Correlacionado con lo anterior, existe poca organización en las comunidades: solo $18 \%$ de las personas que ha vivido en un lugar por más de un año se ha organizado para dar solución a un problema, principalmente sobre servicios públicos. Las razones que expresaron como obstáculos para formar organizaciones fueron que a cada quien le interesan sus propios asuntos, la falta de motivación de ayudar a otras personas y la dificultad para la toma de acuerdos.

Esta problemática es heredada culturalmente a la niñez y se manifiesta a diario en la convivencia escolar, pues las acciones se encaminan hacia el beneficio propio, sin importar qué o a quiénes afecten. Aunado a lo anterior, la visualización que las personas adultas tienen de la niñez, como un sector prospectivo o vulnerable, le impide a la población infantil responsabilizarsede los conflictos y las problemáticas que se presentan en su día a día,lo que da como resultado un desinterés por realizar acciones de transformación en su entorno escolar.

Ante esta necesidad, se implementó un programa que promoviera la participación social en la niñez, basado en la relación entre la responsabilidad y el aumento de la participación en un grupo escolar; para ello se fomentó la conciencia en la niñez acerca de los problemas que 
existen en su escuela y comunidad.Los niños y las niñas realizaron acciones de mejora en su entorno, y con ello se generaron iniciativas y alternativas para el beneficio de todas y todos. Durante su implementación el valor de la responsabilidad se hizo presente en las sesiones para promover el interés por ser personas socialmente responsables. A partir de la implementación se determinó la relación entre la participación y la responsabilidad.

Este estudio está dirigido a docentes o personas interesadas en conocer modelos y teorías de intervención que promuevan la participación infantil social como parte fundamental de una sociedad que ejerce el pleno ejercicio del Estado de derecho.

Con base a lo anterior, la trascendencia de realizar este trabajo consiste en que difícilmente se puede lograr una cultura de emprendimiento social y empresarial para resolver problemas económicos, políticos y sociales desde el trabajo individual, que ocasiona vulnerabilidad en las personas al visualizarse como poco capaces de realizar un cambio significativo en sus intereses. El cambio de percepción de sujetos como seres capaces de transformar su entorno es necesario trabajarlo desde la niñez.

Ante la problemática anteriormente expuesta se promovieron experiencias de organización colectiva que brindan estrategias y herramientas para enfrentar los retos que se tienen como miembro de una sociedad desde temprana edad.

Es necesario tomar en cuenta que la niñez es visualizada de diversas formas en cada grupo social y, por tanto, su participación está determinada por las personas adultas de ese grupo, ante esto, la sociología de la infancia comprende a la niñez como una categoría permanente en las sociedades - aunque sus miembros se renueven constantemente- y como un fenómeno socialmente construido y variable en términos históricos. Básicamente divide el estudio de la niñez en dos grandes perspectivas: la contemporánea y la constructivista (Pavez, 2012). 
La primera perspectiva visualiza a la infancia como una etapa presocial, como producto inacabado o personas que van a ser moldeadas para poderse adaptar a la sociedad, los impulsores de este pensamiento son Parsons, Durkheim, Locke, Rousseau, entre otros.

La segunda perspectiva (Beger y Luckmann, 1968, citado por Pavez, 2012, p.90) considerara al desarrollo y la socialización infantil en diferentes contextos como dinámica; la niñez, al cambiar en un tiempo y un espacio, reconstruye su realidad, por lo que se concibe a la infancia como un fenómeno social.

La diferencia entre estas perspectivas es que la primera posiciona a los niños y las niñas como los próximos sujetos de la sociedad.De esta forma, bajo la condición de vulnerabilidad en la que viven, es necesario transmitir un sin fin de conocimientos básicos para que se adapten a su entorno; en esta primera perspectiva la infancia es concebida como pasiva, mientras que la segunda perspectiva ofrece la oportunidad de reflexionar sobre la niñez como agente activo que transforma su entorno a partir de la reconstrucción que realiza de los significados dados a través del tiempo, es decir que la infancia es un fenómeno en constante cambio.

Los estudios deben enfocarse en las vidas cotidianas de los niños y las niñas,además deben generar investigaciones sobre las realidades macrosociales en las que viven para así tener una visión amplia y un gran mapa del escenario de la infancia,esto con el fin de contextualizar las vidas de las niñas y niños y las y los adolescentes desde su rol individual, respecto a sus formas de participación desde una visión comunitaria o social que incluya posibilidades de asociación y acción colectiva (Corona, 2006).

La participación es un medio que les permite a las personas sentirse capaces de ser autoras de su propia vida y no estar a la merced de otros; permite guiar a la niñez hacia su potencial vinculado a un valor social e individual que tiene como objetivo la organización para promover un buen funcionamiento de la misma organización social. De esta forma, participar 
es tomar parte o compartir a nivel de actitud en determinadas circunstancias.Cabe señalar que la participación es un requisito para el desarrollo de un grupo social (Sánchez, 2000).

Para desenvolverse de forma participativa la confianza se vuelve preciada, al ser un bien supremo para todos y todas, pues permite que los acuerdos tomados entre las personas se cumplan y se trabaje en conjunto. Es difícil poseerla, pero es muy importante para una convivencia armónica.

De esta forma, la intervención educativa hacia la trasformación de espacios exige la sensibilidad del entorno a partir del cuestionamiento permanente y compartido, un cuestionamiento sobre las respuestas dadas, las prácticas modeladas y las categorías e ideologías no cuestionadas, permitiendo una transformación en la mentalidad personal, pues la persona solo está dispuesta a movilizarse si es sensible de su propio potencial y se identifica con diversos proyectos (Garibay y Séguier, 2012).

De esta manera, el papel de la persona docente como co-constructora de aprendizajes que fomenten la participación social debe encaminarse hacia el diálogo, el respeto y la realimentación de las creencias de la niñez; sin embargo, no basta con un profesor o una profesora que tenga estas características, ya que las relaciones sociales deben permitir desafiar, comprometer y cuestionar la realidad de la niñez en el salón de clases tomando en cuenta diversos factores.

Sus elementos principales son la confianza entre las personas y la visualización de la gente como parte de una colectividad, aspectos que se deben fomentar para fortalecer la participación social. Es por ello que su ejercicio colabora a generar grupos menos vulnerables ante adversidades, proactivos en la ejecución de cambios en diversos lugares y corresponsables de lo que acontece en su entorno.

La responsabilidad es un elemento añadido a estos aspectos para que una persona reflexione, administre, se oriente y sea consciente de las consecuencias de sus actos, 
siempre en un plano moral. De esta forma, es un valor esencial en espacios educativos para que los niños y las niñas aprendan a conocer y a vivir con las demás personas, asumiendo deberes y responsabilidades, ya que estos factores conforman las directrices de una institución formadora de miembros proactivos que son capaces de interactuar consigo mismos y con los demás. Por ello la transformación en la visualización de la niñez convierte a las personas en seres capaces de construir cambios en su realidad a partir de la responsabilidad y las acciones de mejora en su entorno.

Por tanto, la participación es un medio que les permite a las personas sentirse capaces de ser autoras de su propia vida, pues guía a la niñez hacia su potencial vinculado al valor social e individual que tiene como objetivo la organización para promover un buen funcionamiento de la convivencia social. Participar es tomar parte o compartir a nivel de actitud en determinadas circunstancias. Cabe señalar que la participación es un requisito para el desarrollo de un grupo social.

De esta forma, la participación social no puede existir sin la convivencia. Sin esta interacción surgen problemas en el intercambio dialógico, por lo que las niñas y los niños deben vivenciar valores consensuados que les permitan una sana convivencia, para así mejorar la calidad de las relaciones sociales e identificar los límites para contextualizar las acciones (Freire, 2009).

Promover la participación social de la niñez en las escuelas significa la formación de personas involucradas con su realidad social. A su vez, esta participación evidencia que las personas inmersas en el contexto como niños y niñas no sólo son capaces de hacer grandes cosas, sino que son actores en la sociedad que aportan soluciones.

A continuación en la figura 1 se presenta la Escalera de la Participación Infantil de Hart (2001), uno de los referentes más utilizados para ubicar el tipo de participación que las niñas y los niños desempeñan en distintos proyectos en su vida cotidiana. Los peldaños inferiores se caracterizan porque el niño o la niña participa pero desconoce la finalidad de esa 
participación; en los siguientes peldaños los niños y las niñas saben por qué realizan las acciones. El gran aporte de este referente es evidenciar la importancia de que la niñez esté informada y sea escuchada en un grupo.

Figura 1. Escalera de la Participación Infantil

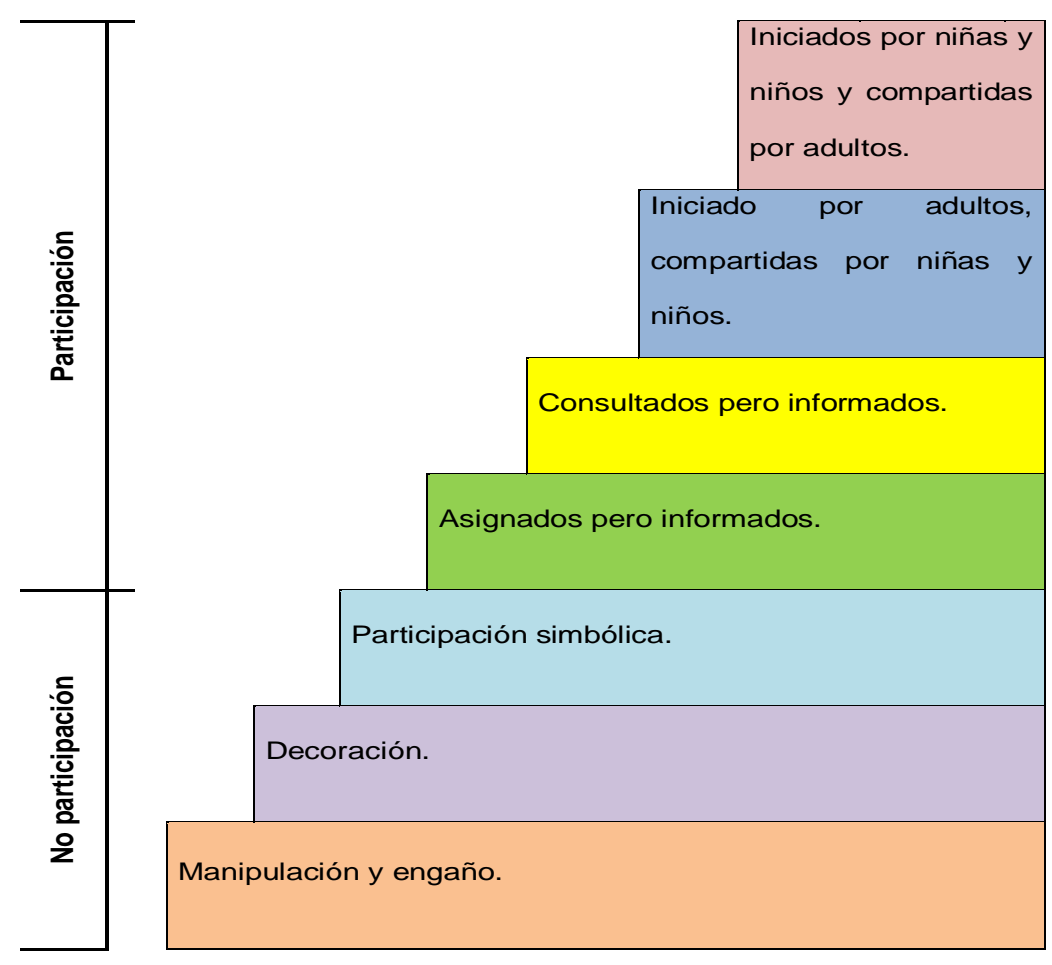

Fuente: Hart, R, 2001

La Escalera de Participación de Hart (2001) es uno de los referentes más importantes en el estado del conocimiento sobre la participación infantil, ya que permite ubicar el tipo de participación que se está potenciando por parte de la niñez. Esta escalera brinda conciencia de la visión que cada persona promotora tiene de las niñas y los niños que intenta potenciar, lo que se pretende lograr y los alcances de la participación. 
El diseño de este estudio se realizó intentando que la niñez se situase en el eslabón más alto de la participación de dicho autor. Su instrumentación se aplicó durante cuatro meses en la Escuela Pública en el Distrito Federal, con la finalidad de mejorar la convivencia escolar fomentando la participación infantil social con el grupo de sexto grado.

En función a la problemática antes destacada fue conveniente plantear la siguiente pregunta: ¿Cómo impacta el fomento de la responsabilidad infantil en la participación social?

Con base en la información sobre las alternativas que existen de participación infantil, el objetivo general es:

- Relacionar el impacto que tiene la responsabilidad con el interés de la niñez por participar en su contexto inmediato a partir de la aplicación de un programa de intervención que fomente la participación infantil.

Asimismo, la hipótesis del estudio es la siguiente: Si las niñas y los niños consideran que es responsabilidad de todos la problemática de su comunidad, tendrán interés por participar activamente en su contexto inmediato para buscar opciones de respuesta a esos problemas.

El instrumento utilizado fue la encuesta, aplicada en dos momentos, al recibir a la población antes del tratamiento (entrada) y una vez terminada la aplicación de las sesiones (salida).Por medio de este se recabó información sobre el grupo para conocer e indagar acerca del comportamiento de la variable dependiente y el de la independiente.

De esta manera, la aplicación del programa de promoción de la participación infantil se llevó a cabo de forma simultánea con las sesiones de reforzamiento del valor de la responsabilidad. Previo al estímulo se administró el tratamiento y después del estímulo se aplicó la prueba (ofreció un referente inicial). Con base en lo anterior, la presente investigación se ubica en el paradigma cuantitativo de tipo pre-experimental, pues el diseño 
se aplicó en un solo grupo y el grado de control fue menor, pues se trata del primer acercamiento al problema de la investigación en la realidad de ese contexto.

La variable independiente (VI) del estudio es la responsabilidad con la comunidad y la variable dependiente (VD) es el interés por seguir participando activamente en su comunidad y posiblemente también en la organización de iniciativas. Lo anterior se esquematiza de la siguiente forma:

Tabla 1. Variables de la investigación

\begin{tabular}{|c|c|c|c|}
\hline Variable & Dimensión & Indicador & \begin{tabular}{|c|} 
Finalidad \\
\end{tabular} \\
\hline $\begin{array}{ll}\text { Nivel } & \text { de } \\
\text { participación } & \text { de } \\
\text { las personas } & \end{array}$ & $\begin{array}{l}\text { Iniciativa de mejorar por } \\
\text { problemas en algún } \\
\text { lugar }\end{array}$ & \begin{tabular}{|lr} 
Propuestas & no \\
asistencialistas & de \\
acción para cambiar \\
algo que no es de su \\
agrado en la \\
comunidad.
\end{tabular} & $\begin{array}{l}\text { Encontrar el grado de } \\
\text { participación que tienen } \\
\text { los niños y las niñas en } \\
\text { su comunidad a partir } \\
\text { de las acciones que } \\
\text { describan. }\end{array}$ \\
\hline \multirow{2}{*}{$\begin{array}{lr}\text { Responsabilidad } \\
\text { con } & \text { su } \\
\text { comunidad } & \end{array}$} & Empoderamiento & $\begin{array}{l}\text { Involucramiento en la } \\
\text { mejora de un espacio }\end{array}$ & $\begin{array}{l}\text { Conocer el rol que } \\
\text { perciben las niñas y los } \\
\text { niños del grado que } \\
\text { tienen en la comunidad. }\end{array}$ \\
\hline & $\begin{array}{l}\text { Conciencia como } \\
\text { persona responsable }\end{array}$ & $\begin{array}{l}\text { Definirse en el mismo } \\
\text { eslabón que las } \\
\text { personas adultas para } \\
\text { resolver problemas }\end{array}$ & $\begin{array}{l}\text { Realización de acciones } \\
\text { en corresponsabilidad } \\
\text { con personas adultas, } \\
\text { niñas y niños }\end{array}$ \\
\hline
\end{tabular}

Fuente: elaboración propia

Con el grupo se aplicaron sesiones que fomentaron la participación infantil a partir de las propuestas que las niñas y los niños realizaron, esto aunado al acompañamiento adulto, mismo que consistió en las siguientes etapas sugeridas en el manual Iniciativas para Compartir(IpC) de Buchbider y Martínez (2011).

“1. Recordatorio Deport-es para Compartir (DpC): El objetivo principal de Deport-es para Compartir fue crear conciencia sobre una realidad global en la población infantil y en la juvenil de una manera inclusiva, sana y divertida, por medio del conocimiento de los ocho Objetivos de Desarrollo del Milenio (ODM). 
2. Sesión pro-iniciativas: En esta sesión las niñas y los niños identificaron problemáticas en su comunidad a través de una serie de preguntas guía que permitieron la reflexión acerca de preocupaciones compartidas sobre su contexto para sintetizar en una preocupación general; es decir, se planteó el tema de interés del colectivo que sirvió como referente para la selección de iniciativas.

3. Diseño del plan de trabajo: En este espacio se seleccionaron las iniciativas para determinar los objetivos y las acciones concretas que realizaría cada equipo para lograrlas. La sesión concluyó con la elaboración de un plan de trabajo.

4. Implementación de iniciativas: En esta etapa se realizaron las acciones planificadas en la sesión de diseño del plan de trabajo con la finalidad de concretar con éxito su iniciativa y mejorar su entorno.

5. Muestra (socialización) de iniciativas: En esta sesión las niñas y los niños invitaron a las madres y los padres de familia, al igual que a otros miembros de la comunidad, a presenciar el trabajo de las iniciativas, la difusión de resultados y el éxito obtenido en el desarrollo de iniciativas con la comunidad".

Estas sesiones surgieron del plan original IpC, pero se realizaron otras sesiones con el fin de observar si eran pertinentes para alcanzar en la población infantil un grado de responsabilidad mayor con su comunidad y así tener mayor interés por seguir participando en su comunidad en posteriores aplicaciones:

- Sesión en valores: En esta sesión las niñas y los niños contextualizaron los Objetivos de Desarrollo del Milenio para mencionar y dibujar espacios en su comunidad donde se realicen acciones apoyando alguno de estos objetivos

- Sesión planea: Se buscó el uso del diálogo y del pensamiento crítico como herramientas indispensables para la comunicación, y de esta forma generar un espacio adecuado para la participación desde la responsabilidad con el otro.

- Manual de niños y niñas: posterior a la etapa de diseño del plan de trabajo se le entregó a cada persona participante un manual donde se enfatizan valores y 
estrategias para lograr una iniciativa con éxito. Esta sesión permitió observar la importancia del cumplimiento de acuerdos para lograr el éxito en la transformación social.

Durante su aplicación se monitoreó el comportamiento de la VD con el incremento del interés por seguir participando. Esta obtuvo un incremento del $6 \%$ al realizar acciones en su contexto; asimismo, la delimitación espacial al terminar la aplicación del programa fue evidente en casi un 33.3\% de las niñas y los niños al elegir escenarios pequeños y acotados, como es el caso de las escuelas y los hogares. De esta forma, las propuestas de acción son más concretas y enfocadas a la realización inmediata de las personas. Lo anterior se esquematiza de la siguiente forma:

Tabla 2. Visualización de personas responsables de generar soluciones y acciones (entrada)

\begin{tabular}{|l|l|c|c|c|}
\hline \multicolumn{1}{|c|}{} & Frecuencia & Porcentaje & Porcentaje válido \\
\hline \multirow{4}{*}{ Válidos } & De cada ser humano & 25 & 83.3 & 83.3 \\
\cline { 2 - 5 } & Del Gobierno & 3 & 10.0 & 10.0 \\
\cline { 2 - 5 } & Otros & 2 & 6.7 & 6.7 \\
\cline { 2 - 5 } & Total & 30 & 100.0 & 100.0 \\
\hline
\end{tabular}

Fuente: elaboración propia

Tabla 3. Visualización de personas responsables de generar soluciones y acciones (salida)

\begin{tabular}{|c|l|c|c|c|}
\hline \multicolumn{2}{|c|}{} & Frecuencia & Porcentaje & Porcentaje válido \\
\hline \multirow{4}{*}{ Válido } & De cada ser humano & 28 & 93.3 & 93.3 \\
\cline { 2 - 5 } & De las personas adultas & 2 & 6.7 & 6.7 \\
\cline { 2 - 5 } & Total & 30 & 100.0 & 100.0 \\
\hline
\end{tabular}

Fuente: elaboración propia 
Tal como se observa, existe un aumento en la responsabilidad por los acontecimientos del mundo en un $10 \%$, en el que los alumnos y las alumnas no visualizaron al Gobierno como el principal ente que resuelve problemas, eso se expresa un empoderamiento de la niñez hacia el crecimiento de la responsabilidad.

Del total de la comunidad infantil encuestada el 93\% considera que los responsables de hacer del mundo un lugar mejor para vivir son las niñas y los niños en colaboración con las personas adultas, y el $7 \%$ opina que solo las personas adultas son las responsables de esta labor. Esto evidencia una actitud favorable ante la responsabilidad.

Las niñas y los niños al finalizar el programa manifestaron que la responsabilidad de hacer algo en su contexto es de cada ser humano. Y en cuanto al interés de seguir participando por medio de iniciativas expresaron lo siguiente:

Tabla 4. Interés por seguir participando con acciones en su medio

\begin{tabular}{|l|c|c|c|c|}
\hline \multicolumn{2}{|c|}{} & Frecuencia & Porcentaje & Porcentaje válido \\
\hline \multirow{3}{*}{ Válidos } & Sí & 26 & 86.7 & 86.7 \\
\cline { 2 - 5 } & No & 4 & 13.3 & 13.3 \\
\cline { 2 - 5 } & Total & 30 & 100.0 & 100.0 \\
\hline
\end{tabular}

Fuente: elaboración propia

En la gráfica anterior se observa que, del total de la comunidad infantil encuestada, casi un $87 \%$ considera que hay alguna otra alternativa para cambiar la situación de la escuela que le agrada, el $13 \%$ restante dijo que no.

A pesar de conocer las dificultades que representa la organización con otras personas para realizar iniciativas, el $87 \%$ tiene interés por seguir generando acciones en su entorno, a diferencia del $13 \%$ que expresa poco interés por realizarlas.Aunque los alumnos y las 
alumnas expresaron un aumento en el interés por resolver problemáticas en su entorno en un $3 \%$, siguen interesados en realizar acciones en la escuela.

\section{Conclusiones}

Es necesario el establecimiento de programas educativos específicos tendientes al fomento de la participación y a una visualización de la niñez como personas que contribuyen a su comunidad, es decir, como seres humanos que se preocupan por una realidad y desarrollan planes de acción para transformar su entorno, ello permite la mejora de la convivencia escolar al corresponsabilizar a todos los actores de lo que acontece en su comunidad.

Con base en los datos estadísticos, el compromiso y las ganas de desarrollar acciones de cambio provienen de las oportunidades de actuación que se brinden a la infancia, acompañadas del fomento de valores que permiten la cooperación en el trabajo actuando responsabilidad. Así, se sugiere que los diseños de programas de participación infantil para la realización de acciones en la comunidad se realicen desde un diseño de sesiones que permita experiencias positivas vinculadas al fomento de los valores anteriormente mencionados para desarrollar habilidades para convivir.

Como dato trascendente no monitoreado y manifestado dentro de las encuestas fue a partir de la aplicación del programa las niñas y los niños cambiaron el escenario en el que les gustaría realizar acciones (de su escuela a su casa), esto muestra a personas más conscientes sobre las implicaciones de la acción al escoger lugares más acotados.

Finalmente, se sugiere en investigaciones futuras diseñar espacios de fomento de estos valores para que haya un reflejo en la responsabilidad, entre niñas, niños y profesores para no sólo brindar la oportunidad de participación sino generarlas junto con niñas y niños. 


\section{Referencias}

Buchbinder, D. y Martínez, C. (2011). Iniciativas para Compartir. México: AMNU.

Chávez, C. (2006). Participación social: retos y perspectivas. México: UNAM.

Corona, Y. (2006).Desarollos conceptuales sobre ciudadanía e infancia. Ciudadania y poder. Recuperado de: http://web.uned.ac.cr/revistas/index.php/caes/about/submissions\#authorGuidelines

Freire, P. (2009). Teoría de la autonomía. España: Siglo XXI.

Garibay, F. y Séguier, M. (2012). Pegagogía y prácticas enmacipadoras. México: Instituto Politécnico Nacional.

Hart, R. (2001). La participación de los niños en el desarrollo sostenible. Barcelona: UNICEF.

Pavez, I. (2012). Sociología de la Infancia: las niñas y los niños como actores sociales. Revista de sociología, 27, 81-102.

Sánchez, M. A. (2000). La participación. Metodología y práctica. Madrid: Popular

SEDESOL (2007). Encuesta de capital social urbano 2006. Recuperado de: http://www.infodf.org.mx/iaipdf/extra/doctos/torre.pdf.

World Values Survey (2005). Download WVS 2005 Questionnaire. Recuperado de: http://www.worldvaluessurvey.org/wvs/articles/folder_published/survey_2005 